\title{
AMPLIACIÓN DE LA FRONTERA AGROPECUARIA CHAQUEÑA: EL OESTE CHAQUEÑO Y EL CULTIVO ALGODONERO
}

\author{
PROF. MGR. VIVIANA CLAUDIA PERTILE (*) \\ Departamento de Geografía \\ Facultad de Humanidades \\ Universidad Nacional del Nordeste (Argentina) \\ Avenida Las Heras $N^{\circ} 727-$ \\ 3500 - Resistencia - Chaco - Argentina Teléfono/ \\ FAX: $0054-3722-446958$
}

\section{Resumen}

El planteo fundamental del presente trabajo se centra en un fenómeno que se está produciendo en los últimos años en el sector agrícola del espacio objeto de estudio, según lo indican los datos del CENSO NACIONAL AGROPECUARIO 1988, es la expansión de la frontera agropecuaria, desarrollándose desde los tradicionales núcleos algodoneros de la planicie centrochaqueña, hacia el occidente, alcanzando el área de secano de la provincia de Santiago del Estero donde, además, comienzan a observarse cambios en la estructura productiva y en el tamaño de las explotaciones agropecuarias. Un elemento a tener en cuenta en la ampliación de dicha frontera lo constituyen, sin lugar a dudas, las precipitaciones, éstas con sus variaciones interanual y en sentido Este-Oeste determinan que el agua sea el elemento limitante en las prácticas agropecuarias del Oeste chaqueño y Este santiagueño. Es intención de este trabajo, indagar acerca de la incorporación de nuevas superficies a la actividad agrícola en el Sudoeste Chaqueño y Oriente Santiagueño.

\section{Palabras claves}

$<$ Frontera $><$ Agropecuaria $><$ Actividades Agropecuarias $><$ Diversificación Agrícola $>$

\section{EXPANSION OF THE CHAQUEÑEAN AGRICULTURAL FRONTIER: THE WEST OF CHACO AND THE COTTON CROPS}

The basic issue of this paper is a phenomenon that has been taking place during the last years in the agricultural area and which is the object of this study, that is the expansion of the agricultural frontiers according to the Agricultural National Census of 1988. This expansion springs from the traditional cotton nuclei in the mid-Chaquenean plains and spreads towards the West reaching the dry lands of the province Santiago del Estero, where some changes have been observed in the productive structure en in the volume of agricultural exports. An aspect to be considered in the expansion of this frontier is the rain regime with it annual and Eat-West variations which determines limit the agricultural practices in Western Chaco and Eastern Santiago del Estero. It is the purpose of this paper to inquire about the incorporation of new lands to the agricultural activities in South-western Chaco and Eastern Santiago del Estero.

Key word:

$<$ Agricultural Frontier $><$ Agricultural Activities $><$ Agricultural Diversification $>$ 


\section{AMPLIACIÓN DE LA FRONTERA AGROPECUARIA CHAQUEÑA: EL OESTE CHAQUEÑO Y EL CULTIVO ALGODONERO}

\section{INTRODUCCIÓN}

Si tenemos en cuenta la composición de la estructura económica primaria de la Provincia del Chaco, podemos afirmar que ésta se divide básicamente en dos subsectores: el agrícola y el ganadero. Esta actividad agropecuaria a nivel provincial participa en promedio con el 16\% del P.B.G. dentro de la cual la agricultura ocupa el primer lugar con el $65 \%$, seguido por la ganadería con un $24 \%$ y en tercer lugar se encuentra la actividad forestal con el $6 \%$ del P.B.G agropecuario (GELMAN,1995), reducida en los últimos años como consecuencia de la disminución de la explotación del monte nativo. Respecto al resto de las actividades como la silvicultura, la pesca y los servicios agrícolas se puede decir que sus participaciones en la generación del P.B.G. provincial son reducidas.

En la actualidad la base de la estructura productiva agropecuaria está dada por el cultivo del algodón, primacía que por décadas, caracterizó la base económica de la región. Por otra parte, desempeña un rol estratégico en las economías regionales del Norte Argentino y en el desarrollo de la industria textil nacional. El auge de las prácticas culturales en los llamados núcleos algodoneros localizados en territorio chaqueño, formoseño, santiagueño y santafecino, responden a una variada gama de causas entre las que se destaca el factor humano, que imprimió ciertas características en la estructura y parcelamiento agrario, en cada caso de un modo particular; y un medio natural cuyas variables térmica y pluviométrica aportan las condiciones, sino óptimas, al menos adecuadas para el desarrollo del ciclo vegetativo del mismo.

El cultivo del algodón representa por lo tanto un aporte muy importante al Producto Bruto Agrícola (PBA) de las provincias del Nordeste Argentino (fundamentalmente en Chaco y Formosa). De acuerdo a datos de la Secretaría de Agricultura, Ganadería, Pesca y Alimentación (SAGPyA), más de 35.000 agricultores (del NEA) realizan en la actualidad esta actividad productiva, lo que refuerza la idea de su relevancia económica y social, (INTA, 1999).

Sin dudas, en el ámbito provincial, la planicie centrochaqueña ha sido desde siempre el área algodonera por excelencia alrededor de la cual se hacía el monocultivo (Quitilipi - Sáenz Peña - Villa Angela y Las Breñas) coincidiendo con el espacio donde los montos pluviométricos oscilan entre los 700 y $1000 \mathrm{~mm}$ anuales, degradándose dicha área hacia el occidente, fundamentalmente a causa del incremento de la aridez. Esta situación ha ido cambiando en los últimos años, como consecuencia del aumento de las lluvias en la región.

Desde un principio se definió claramente la principal cuenca algodonera sobre la planicie centrochaqueña: el Domo Algodonero, con polos en Sáenz Peña, Quitilipi; Las Breñas; Charata, Gral. Pinedo y Villa Angela, en las cuales las explotaciones oscilaban alrededor de 30 hectáreas prolongándose tenuemente en el oriente de Santiago del Estero (BRUNIARD, 1975-78:77). 
El Objetivo de este trabajo es indagar acerca de la incorporación de nuevas superficies a la actividad agrícola en el Sudoeste Chaqueño y su prolongación hacia el Oriente Santiagueño a partir del corrimiento de la isohieta de 800 milímetros.

\section{EXPANSIÓN DEL ÁREA ALGODONERA CHAQUEÑA}

Desde hace varios años la Argentina ocupa uno de los primeros lugares en la expansión y producción algodonera mundial, y ha desarrollado ventajas competitivas que le permitieron destacarse por las ventas externas, sobre todo a los países miembros del Mercosur.

Varios han sido los factores que determinaron la ampliación del área algodonera, particularmente en la región que nos ocupa -el Sudoeste chaqueño-, entre ellos podemos mencionar por ejemplo al aumento de las precipitaciones (corrimiento de la isohieta de 800 milímetros) las cuales permitieron sembrar en áreas que antes solo se podían lograr bajo riego; la reducción de los precios de los productos químicos, la implementación de tecnología y dentro de ella la mecanización de la cosecha; este incremento en la extensión del área algodonera ha trascendido los límites del Chaco y abarcó ciertos departamentos del Este de la provincia de Santiago del Estero.

\section{Corrimiento De La Isohieta De 800 Milímetros}

La distribución espacial de los montos pluviométricos en el territorio provincial experimentaron variaciones importantes en las últimas tres décadas. Las lluvias en el Oriente Chaqueño se mantenían hasta la década del ' 50 con valores promedios de 1000 a $1200 \mathrm{~mm}^{1}$. A partir de la década del '60 comienzan dichas oscilaciones que determinaron períodos más húmedos y que dieron lugar al incremento de las precipitaciones.

Este comportamiento de las precipitaciones lo podemos ver a partir de las siguientes representaciones, realizadas mediante el análisis de las Estadísticas Climatológicas, proporcionadas por la Fuerza Aérea Argentina, para cada uno de los períodos considerados (1961-70; 1971-80 y 1981-90). De esta manera fue posible trazar las isohietas y observar el corrimiento al que hacemos referencia.

\footnotetext{
1 En atlas Climático de la República Argentina, del Servicio Meteorológico Nacional, se puede observar la distribución de las precipitaciones medias anuales correspondientes al período 1921-1950, en el territorio nacional; allí el oriente Chaqueño se encuentra perfectamente delimitado por las isohietas de 1000 y $1200 \mathrm{~mm}$.
} 

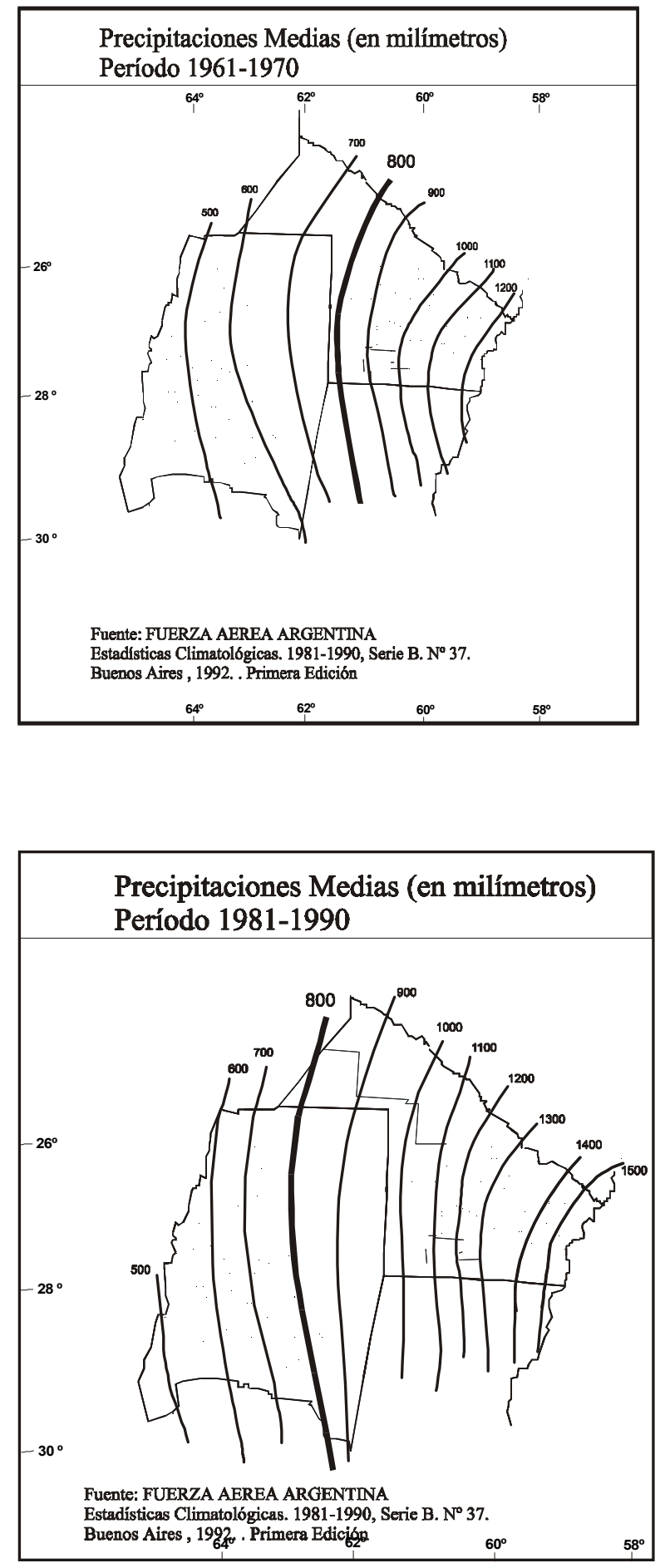

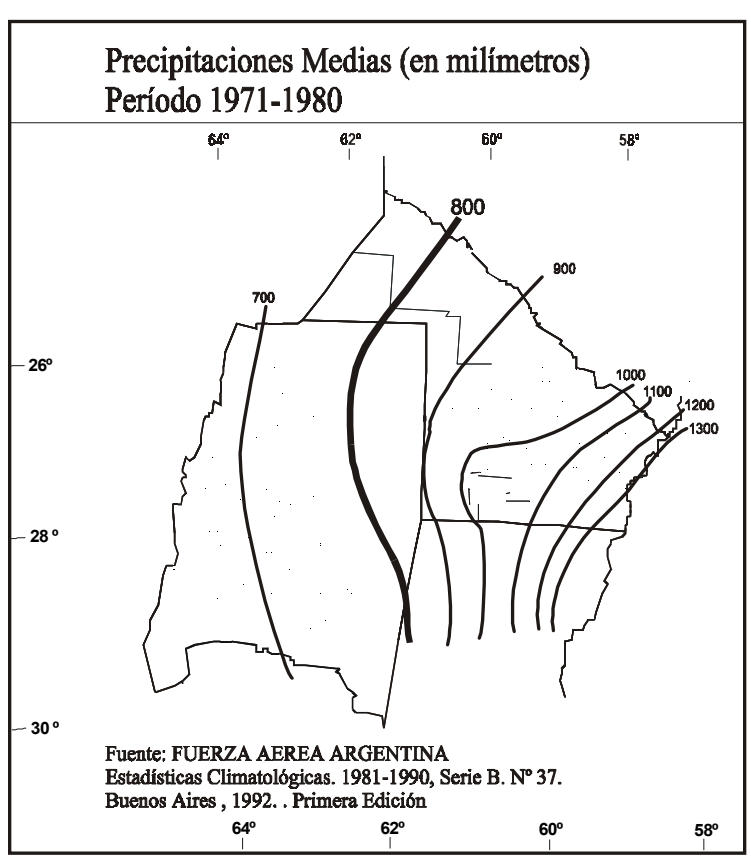

Se puede observar que en el período 1961-70 la isohieta de $800 \mathrm{~mm}$ atravesaba el Oeste de la provincia, próxima al límite con Santiago del Estero; en el período 1971-80 se corre hacia el occidente, pasando por los departamentos de Copo, Alberdi, Moreno y Felipe Ibarra; en el último período, la isohieta se encuentra aún más hacia el Oeste, atravesando los Dptos. Del Sur de Santiago, como Mitre, Salavina, Aguirre, Avellanda.

Este incremento de las precipitaciones permitió la incorporación de tierras al cultivo del algodón en el sudoeste chaqueño. A modo de ejemplo presentamos algunos datos estadísticos proporcionadas por el Ministerio de la Producción de la Provincia del Chaco, que permiten corroborar la expansión de las superficies cultivadas hacia el sudoeste. 
Superficie Cultivada Y Producción De Algodón En El Centro Y Sudoeste Chaqueño Entre Las Campañas Agrícolas 1983-84 Y 1995-96.

\begin{tabular}{|c|c|c|c|c|c|c|c|}
\hline CENTRO & 1983-84 & 1984-85 & 1985-86 & 1986-87 & $1987-88$ & 1988-89 & 1989-90 \\
\hline SUP.CULT. (has) & 188500 & 174500 & 129000 & 81500 & 170000 & 171000 & 174000 \\
\hline PROD. (ton.) & 245400 & 202600 & 123300 & 96550 & 302382 & 191600 & 305220 \\
\hline & 1990-91 & 1991-92 & $1992-93$ & 1993-94 & 1994-95 & 1995-96 & \\
\hline SUP.CULT. (has.) & 250000 & 214500 & 151100 & 179000 & 256500 & 309500 & \\
\hline PROD. (ton.) & 356115 & 217100 & 191258 & 258820 & 328722 & 408810 & \\
\hline
\end{tabular}

\begin{tabular}{|c|c|c|c|c|c|c|c|}
\hline SUDOESTE & $1983-84$ & 1984-85 & 1985-86 & 1986-87 & 1987-88 & 1988-89 & $1989-90$ \\
\hline SUP.CULT. (has.) & 45200 & 43500 & 33000 & 22000 & 55000 & 53700 & 62500 \\
\hline PROD. (ton.) & 59500 & 52500 & 32100 & 27000 & 98540 & 60770 & 108105 \\
\hline & 1990-91 & 1991-92 & $1992-93$ & 1993-94 & 1994-95 & 1995-96 & \\
\hline SUP.CULT. (has.) & 97000 & 83000 & 62000 & 97000 & 151000 & 186000 & \\
\hline PROD. (ton.) & 140710 & 81100 & 83790 & 147375 & 227618 & 269770 & \\
\hline
\end{tabular}

Fuente: Elaboración propia con datos proporcionados por: M.G.A. -CHACO-. Dirección de Planificación. Ministerio de la Producción.

El área Central abarca los siguientes departamentos: Comandante Fernández, Maipú, Independencia, Mayor Luis J. Fontana, O'Higgins, Pcia. de la Plaza, Quitilipi, San Lorenzo y 25 de Mayo, en tanto que el Sudoeste comprende: 9 de Julio, Chacabuco, 12 de Octubre, Fray J. Santa. María de Oro, General Belgrano y 2 de Abril.

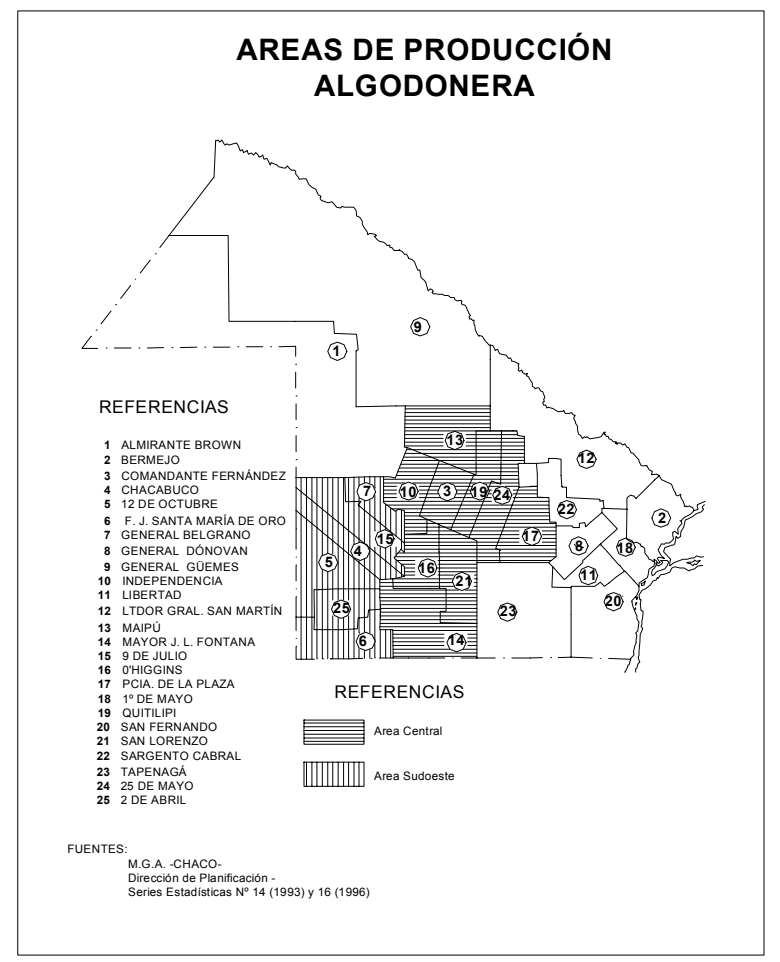


En el área central de la provincia se observa una situación bastante regular con un pequeño incremento en los últimos años.

Como lo dijéramos en párrafos anteriores, otro de los factores que determinaron la expansión de la frontera agropecuaria, fue la aplicación de la tecnología. La producción de las pequeñas explotaciones se volvieron insuficientes para hacer económicamente rentable la actividad, razón por la cual se vio la necesidad de contar con superficies más grandes para producir en escala, bajar los costos y aumentar la rentabilidad. Esto hizo que la mecanización cosecha se convirtiera en la protagonista de ello, permitiendo la recolección de una producción considerablemente más importante. Durante los años noventa, la mecanización de la recolección ha seguido un ritmo acelerado, casi $90 \%$ de la producción total se recolecta a máquina. Las máquinas recolectoras en su mayoría son importadas de los EE.UU, pero además existen las fabricadas en Argentina, desarrolladas a partir de un prototipo diseñado por INTA; se dice que las nacionales son las más idóneas, por que se adaptan a la altura de las plantas locales -la altura de las plantas en nuestro país son más bajas, debido a la carencia de fertilizantes nitrogenados y en algunos casos falta de agua.

Este proceso de expansión se caracterizó por: * la presencia de grandes productores; * el surgimiento de desmotadoras, preferentemente privadas y la pérdida de fuerza de las cooperativas; * pérdida de competitividad de la pequeña y mediana empresa; * etc.

Con relación al tamaño de las explotaciones algodoneras en nuestra provincia, podemos decir, según datos proporcionados por los últimos Censos Nacionales Agropecuarios (19601988), que hubieron variaciones, y que en los últimos años prosperaron los grandes productores agrícolas.

Tamaño de las explotaciones agropecuarias en la provincia del Chaco

\begin{tabular}{|c|c|c|c|}
\hline \multicolumn{4}{|c|}{ Censo Agropecuario de 1960} \\
\hline Tamaño & $\begin{array}{l}\mathbf{N}^{\circ} \text { de } \\
\text { Explot. }\end{array}$ & $\%$ & Superficie \\
\hline 0 - 5 has & 1762 & 6.6 & 5888 \\
\hline $5,1-25$ has & 6188 & 23.0 & 99321 \\
\hline $25,1-100$ has & 13599 & 50.6 & 945129 \\
\hline Hasta 100 has & & 80.2 & \\
\hline $100,1-200$ has & 2362 & 8.8 & 366946 \\
\hline $200,1-400$ has & 845 & 3.1 & 242723 \\
\hline $400,1-1000$ has & 665 & 2.5 & 421402 \\
\hline $1000,1-2500$ has & 616 & 2.3 & 1115630 \\
\hline $2500,1-5000$ has & 132 & 0.5 & 490627 \\
\hline $5000,1-10000$ has & 50 & 0.2 & 372963 \\
\hline Más de 10000 has & 30 & 0.1 & 995243 \\
\hline S/ Determinar & 604 & $|2.2|$ & ----- \\
\hline
\end{tabular}

\begin{tabular}{|l|c|c|c|}
\hline \multicolumn{5}{|c|}{ Censo Agropecuario de 1988} \\
\hline Tamaño & $\begin{array}{l}\mathbf{N}^{\mathbf{0}} \text { de } \\
\text { Explot. }\end{array}$ & $\mathbf{\%}$ & Superficie \\
\hline 0 - 5 has & 1147 & $\mathbf{6 . 5}$ & 3808 \\
\hline $5,1-25$ has & 2333 & $\mathbf{1 3 . 3}$ & 35805 \\
\hline 25,1-100 has & 6355 & $\mathbf{3 6 . 1}$ & 429848 \\
\hline Hasta 100 has & & $\mathbf{5 5 . 9}$ & \\
\hline 100,1-200 has & 3076 & $\mathbf{1 7 . 5}$ & 466343 \\
\hline 200,1-400 has & 2690 & $\mathbf{1 5 . 3}$ & 860023 \\
\hline 400,1-1000 has & 1035 & $\mathbf{5 . 9}$ & 720249 \\
\hline 1000,1-2500 has & 691 & $\mathbf{3 . 9}$ & 1111812 \\
\hline 2500,1-5000 has & 172 & $\mathbf{1 . 0}$ & 605843 \\
\hline Más de 5000 has & 96 & $\mathbf{0 . 5}$ & 1090887 \\
\hline
\end{tabular}


Estos datos muestran la disminución de las pequeñas explotaciones (hasta 100 has) de un $80,2 \%$ a un $55 \%$; en contraposición el incremento del tamaño de las explotaciones agropecuarias de mas de 100 has en entre los años 1960 y 1988.

Una consecuencia negativa desde el punto de vista social y económico de la implementación de las cosechadoras, es que ha reducido notablemente la utilización de la recolección manual, en consecuencia disminuyó el desplazamiento o traslado de los cosecheros (entre $60 \mathrm{y}$ $70.000)^{2}$, con lo cual el circuito económico se ve interrumpido en su normal desenvolvimiento, por cuanto restringe el consumo en general.

El siguiente gráfico nos muestra lo sucedido con la evolución del cultivo de algodón en la provincia del Chaco en la década del '90.

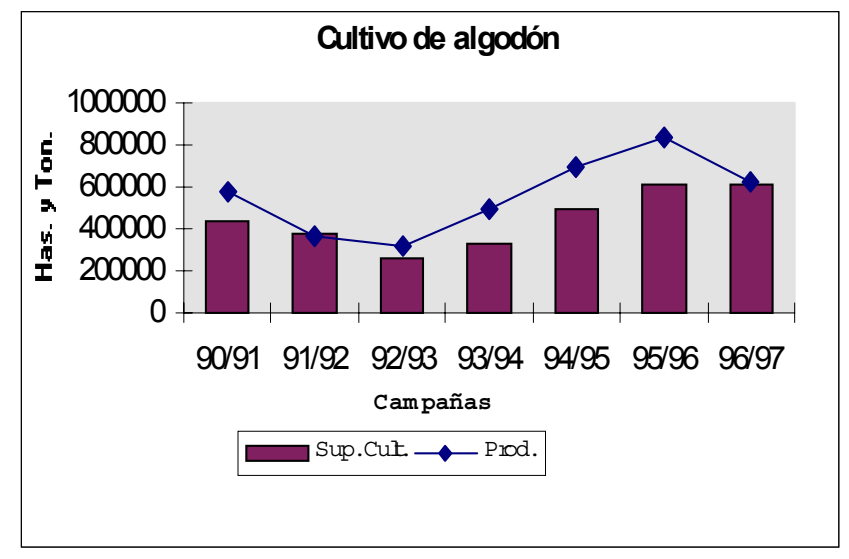

Observamos que en los primeros años de la década las superficies dedicadas al cultivo experimentaron una tendencia decreciente, registrándose una pronunciada baja en la campaña 1992-93, consecuencia de la importante disminución en el precio internacional del algodón, coincidiendo además, con condiciones climáticas poco favorables.

A partir de la temporada 1993-94 esa tendencia se revirtió, llegando a la campaña 1995-96 con el récord histórico hasta ese momento.

\section{Campaña 1995-96 - Récord Histórico}

Esta campaña representó un récord histórico nacional. Los factores que influyeron fueron varios, el más importante fue sin dudas el precio alcanzado por el producto en la campaña anterior, (los índices de precios nacionales fueron más elevados que los internacionales). Otro factor fue la disminución de los costos de insumos y de las maquinarias agrícolas, tal como lo manifestáramos anteriormente, las maquinarias podían ser adquiridas a valores inferiores a los de la década del '80, constituyéndose en otro de los determinantes para que los productores ampliaran las superficies dedicadas al cultivo ICAC RECORDER (Op. Cit.)

Es importante destacar que esta campaña no sólo representó un récord histórico, sino que también determinó un cambio en los primeros puestos provinciales en lo que concierne a la producción.

\footnotetext{
${ }^{2}$ Los cosecheros no solo realizaban la cosecha manual del algodón, sino también otras actividades relacionadas con el laboreo y cuidado del cultivo como ser -las carpidas- hoy reemplazadas por el uso de agroquímicos desmalezadores.
} 
Históricamente las primeras cinco provincias productoras de algodón fueron: Chaco, Formosa, Santa Fe, Santiago del Estero y Corrientes. A partir de la campaña 1995-96 la situación cambió, Santiago del Estero se convierte en segunda productora, después de Chaco; Salta tercera y Corrientes dejó de estar entre las primeras cinco productoras

\section{Campaña agrícola 1995-96.}

\begin{tabular}{|l|l|l|l|c|}
\hline Provincia & $\begin{array}{l}\text { Superficie } \\
\text { Sembrada }\end{array}$ & $\begin{array}{l}\text { Superficie } \\
\text { Cosechada }\end{array}$ & $\begin{array}{l}\text { Producción } \\
\text { Kg. }\end{array}$ & Rendimiento kg/ha. \\
\hline Chaco & 613500 & 594300 & 832020 & 1400 \\
\hline S. del Estero & 214000 & 210400 & 283560 & 1347 \\
\hline Formosa & 55000 & 47600 & 47124 & 990 \\
\hline Santa Fe & 54700 & 54600 & 85064 & 1557 \\
\hline Otras & 71450 & 60310 & 77714 & 1280 \\
\hline Total del País & 1008650 & 967210 & 1325485 & 1370 \\
\hline
\end{tabular}

Podemos manifestar que este protagonismo que tiene Santiago del Estero a partir de la campaña agrícola 1995-96 con su producción de algodón, se enmarca también dentro del aspecto central de nuestro trabajo (Ampliación de la frontera agrícola Chaqueña). Si tenemos en cuenta el monto y la distribución de las lluvias de la última década, podemos afirmar que éstas favorecieron el desarrollo del cultivo de algodón en el Este de Santiago del Estero. Este aumento está asociado a la expansión y desarrollo de productores del centro chaqueño, que por iniciativa propia, trasladaron herramientas y trabajo a campos cercanos a la frontera santiagueña, para luego atravesarla y a través del arrendamiento y/o la compra, experimentar un importante crecimiento de las superficies cultivadas. Tomando como referencia la campaña 1993-94, en la provincia de Santiago del Estero se sembraron 65.300 hectáreas para alcanzar en 1997-98 las 253.700 hectáreas, según datos proporcionados por la Secretaría de Agricultura, Ganadería, Pesca y Alimentación del año 1998. Este avance algodonero de los agricultores chaqueños, tuvo en su primer etapa el carácter de Asociación, por cuanto, varios productores aunaron esfuerzos, capital y maquinarias, para aumentar la extensión de las superficies sembradas. En la zona central de la provincia del Chaco eran pocas las explotaciones que excedían las 100 hectáreas, éstas superficie considerada insuficiente ya que no permiten alcanzar grados de rentabilidad significativos, considerando los costos que deben cubrirse y la competitividad existente.

Es preciso aclarar que en esta ampliación de las superficies dedicadas al cultivo del algodón, también tuvo su cuota protagónica la provincia de Santa Fe, especialmente en los departamentos del norte de la provincia (9 de Julio, Obligado y Vera), donde la superficie cultivada en la campaña 1992-93 alcanzó las 19.500 has. y en 1996-97 superaron las 50.000 hectáreas.

\begin{tabular}{|c|c|c|c|c|c|c|c|c|}
\hline \multirow[b]{2}{*}{ Provincia } & \multicolumn{2}{|c|}{$1993 / 1994$} & \multicolumn{2}{|c|}{$1994 / 1995$} & \multicolumn{2}{|c|}{$1995 / 1996$} & \multicolumn{2}{|c|}{$1996 / 1997$} \\
\hline & $\begin{array}{r}\text { Sup. Cult. } \\
\text { en Has. }\end{array}$ & \begin{tabular}{|l|} 
Producción \\
miles de ton.
\end{tabular} & $\begin{array}{l}\text { Sup. Cult. } \\
\text { en Has. }\end{array}$ & \begin{tabular}{|l|} 
Producción \\
miles de ton.
\end{tabular} & $\begin{array}{r}\text { Sup. Cult. } \\
\text { en Has. }\end{array}$ & $\begin{array}{l}\text { Producción } \\
\text { miles de ton. }\end{array}$ & $\begin{array}{l}\text { Sup. Cult. } \\
\text { en Has. }\end{array}$ & \begin{tabular}{|l|} 
Producción \\
miles de ton.
\end{tabular} \\
\hline Chaco & 335.500 & 491.600 & 498.000 & 705.300 & 609.900 & 832.010 & 612.000 & 622.700 \\
\hline Santiago del E. & 65.300 & 85.400 & 140.700 & 227.200 & 216.100 & 309.440 & 218.300 & 231.990 \\
\hline Santa Fe & 34.000 & 39.100 & 35.000 & 51.800 & 54.700 & 85.460 & 37.500 & 52.800 \\
\hline Total Argentina & 503.610 & 705.780 & 735.400 & 1.073 .347 & 1.005 .300 & 1.347 .040 & 960.060 & 1.029 .866 \\
\hline
\end{tabular}

Fuente: Elaboración propia en base a datos proporcionados por la SAGPy A . En Diario Norte, Resistencia, Chaco, 17junio de 1998. 
Alejandro Valeiro ${ }^{3}$ en un informe publicado en Noticias INTA, en la Página Web INTA manifestaba lo siguiente: "el desmote de algodón en nuestro país, lejos de tender a constituirse en un simple servicio al productor (como ocurre por ejemplo en Estados Unidos), ha asumido un rol de liderazgo en la expansión. Las empresas crecen-además de la capacidad de desmote- en su actividad de financiamiento de la producción, no sólo del capital circulante en una campaña, sino incluso de grandes inversiones como son las cosechadoras. Por otra parte, y como consecuencia de la privatización del los Bancos provinciales del Chaco y de Santiago del Estero y las restricciones a los créditos bancarios, el financiamiento de la producción se ha convertido en la clave del complejo agroindustrial algodonero, siendo las empresas desmotadoras privadas de mayor envergadura las que acceden al crédito de los compradores externos, con tasas de intereses sensiblemente inferiores a las locales, lo que determina una posición privilegiada dentro del complejo.

En lo que respecta a la capacidad de desmote, la provincia del Chaco ha visto incrementar sus toneladas desmotadas; entre los años 1992 y 1997, según datos publicados por en Norte Rural, del 17 de junio de 1998, el Chaco alcanzó las 735 mil toneladas desmotadas, de las cuales Presidencia Roque Sáenz Peña lideró la radicación industrial textil con una capacidad de 132 mil toneladas, el segundo y tercer lugar en lo provincia lo ocupan las localidades de Villa Angela y Las Breñas con 78 y 68 mil toneladas, respectivamente; el segundo lugar a nivel nacional lo ocupa Santa Fe con 178 mil toneladas; y el tercer lugar, Santiago del Estero con 128 mil toneladas; luego se sitúan las provincias de Salta, Formosa, Catamarca, etc.

La capacidad de desmote en la Argentina es de 1.210 mil toneladas, de los cuales el 83,3\% lo cubren Chaco, Santa Fe y Santiago del Estero y el 14,7\% las provincias restantes. Estas 1210 mil toneladas desmotadas se repartían en año 1998 en 126 desmotadoras, 82 de ellas se localizan en Chaco; de esas 30 son Cooperativas y 96 son privadas.

Según las estadísticas proporcionadas por le Departamento de Estimaciones agrícolas del Ministerio de Agricultura y Ganadería de la Nación, en el año 1973 existían en el país 136 desmotadoras de las cuales 41 correspondían a Cooperativas y 95 eran privadas.

Número de desmotadoras

\begin{tabular}{|l|c|c|c|c|}
\hline Desmotadoras & \multicolumn{2}{|c|}{1973} & \multicolumn{2}{c|}{1998} \\
\hline Cooperativas & 41 & $30 \%$ & 30 & $24 \%$ \\
\hline Privadas & 95 & $70 \%$ & 96 & $76 \%$ \\
\hline TOTAL & 136 & $100 \%$ & 126 & $100 \%$ \\
\hline
\end{tabular}

Este cuadro nos muestra que las desmotadoras privadas aumentaron proporcionalmente respecto del total y disminuyeron las cooperativas.

A partir de la información recopilada y a los efectos de corroborar la información presentada, se han elaborado algunos mapas que sintetizan lo expresado:

- Mapa $\mathrm{N}^{\circ} 1$ "Superficie Sembrada con algodón en Hectáreas. Este mapa muestra la evolución de la superficie cultivada del cultivo del algodón en dos décadas: 70 y 90,. De cada década se tomaron cuatro campañas y se obtuvo un promedio, el resultado fue

\footnotetext{
${ }^{3}$ Ing. Agr. Coordinador del Area de Desarrollo Rural del INTA Santiago del Estero.
} 
volcado mediante gráficos de barras -una barra para cada período, con idénticas escalas verticales- en cada uno de los departamentos de la provincia del Chaco, Santiago del Estero y Santa Fe. El resultado evidencia un notable crecimiento fácilmente observable en las alturas de las barras del período correspondiente a la década del ' 90 .

La misma información estadística fue utilizada para relacionarla con la superficie departamental. De esta manera se obtuvo un dato que revela la importancia que tiene el cultivo de algodón para cada uno de los departamentos de estas tres provincias. Al mismo tiempo hemos podido también plasmar cartográficamente la ampliación de las superficies algodoneras.

- Mapa $\mathrm{N}^{\mathrm{o}} 2$ y 3, muestran la evolución de las superficies cultivadas en relación con la superficie departamental, en proporción porcentual en las décadas del 70 y 90, es evidente el crecimiento de las superficies cultivadas entre una y otra década.

\section{CONCLUSIÓN}

En los últimos años de la década del 90' se pudo advertir en la provincia del Chaco una significativa inversión en el ámbito algodonero. Si bien la superficie cultivada a principios de ésta década experimentó tendencias decrecientes, como consecuencia de la importante disminución del precio del textil, es a mediados de la misma cuando se produce la expansión del área algodonera con el consiguiente incremento de la producción.

Hasta principios de los años 80' la mayor parte de la superficie dedicada a éste cultivo se hallaba en los departamentos O'Higgins, Mayor L. J. Fontana, Independencia, C. Fernández, Quitilipi y 25 de Mayo, área conocida como el corazón algodonero de la provincia, pero dicho corazón o núcleo se desplazó hacia el Sudoeste en los últimos años, traspasando el límite chaqueño, ya que campos del Este santiagueño han sido también ocupados por el textil; es preciso destacar que la mayoría de ellos pertenecen a productores chaqueños, quienes buscaron ampliar sus explotaciones en las áreas contiguas a la provincia del Chaco, favorecidos por buenas siembras, aumento de las lluvias, tamaño de las explotaciones facilidades de ocupación, etc.

El reconocimiento directo en la zona de estudio permitió observar que, gran parte de las tierras que hace algunos años atrás estuvieran ocupadas por el monte chaqueño, se encuentra hoy ocupada por el noble algodón, cultivo que a pesar de experimentar importantes variaciones en su producción, continúa siendo económicamente el más significativo en la provincia del $\mathrm{Chaco}$, por cuanto conserva la primacía en la estructura productiva primaria y es a la vez el cultivo que más expectativas despierta en los productores.

En relación a esto último, debemos considerar las menores exigencias climáticas que posee el algodón respecto de otros cultivos, a los que podríamos añadir el incremento de las precipitaciones hacia el Oeste, fenómeno que en la última década pudo ser advertido según las estadísticas pluviométricas analizadas.

Es posible deducir que otra de las razones por la que el polo de desarrollo algodonero se ha desplazado hacia el SO. en la provincia del Chaco y E. y NE. de Santiago del Estero, se debió a la necesidad de poder realizar explotaciones en gran escala, mecanizadas e extensivas, para lograr de esa manera mejor rentabilidad, por cuanto es muy difícil sobrevivir con 40 o 60 has. -extensión media de las explotaciones algodoneras- con lo costos actuales y con la competitividad y rentabilidad existente.

La presencia de un gran número de desmotadoras privadas localizadas a lo largo de las Rutas Provinciales $\mathrm{N}^{\circ} 16,94$ y 95, nos habla a las claras de las esperanzas depositadas en el algodón. En el año 1998 se desmotaron en la Argentina 1210 mil toneladas de fibra, correspondiéndole al Chaco 750 mil toneladas, esto es el $61,9 \%$ del total desmotado en el país. 
En definitiva, hay que destacar que la expansión hacia el SO. Chaqueño y E. santiagueño se produce porque se conjugan una serie de condiciones: climáticas, humanas y de mercado son propicias, a pesar de que en ciertas ocasiones los magros rendimientos obtenidos no permitieron lograr márgenes para saldar las cuentas de algunos productores, ocasionando en muchos casos la quiebra económica y el abandono de la actividad.

En síntesis, podemos concluir que este proceso de expansión agrícola reúne las siguientes características:

- La expansión de la frontera agrícola hacia el Occidente: esto es, la incorporación de nuevas tierras del Suroeste Chaqueño y Este Santiagueño al circuito productivo agropecuario;

- La presencia de grandes productores, algunos de los cuales tienen entre mil y cinco mil hectáreas dedicadas a la agricultura, pero también es preciso mencionar la existencia de megaproducciones que alcanzan las 10 mil hectáreas.

- Multiplicación de las desmotadoras privadas en Chaco y Santiago del Estero, cuya capacidad de desmote cubría en el año 1998 el 83\% del total del país, la contrapartida de este hecho lo constituye el deterioro económico de las Cooperativas y Pymes.

- La tecnificación agrícola, representada fundamentalmente por la cosecha mecánica, gracias al abaratamiento de las maquinarias nacionales e importadas. En este punto, debemos mencionar consecuencias negativas desde el punto de vista social y económico por cuanto no sólo se han reducido notablemente los desplazamientos de los cosecheros, con las implicancias que ello genera en el circuito económico general, sino también que miles de ellos quedaron sin trabajo al desaparecer prácticamente la cosecha manual.

- Desaparición del pequeño productor del circuito productivo, ante la imposibilidad competitiva y de lograr rentabilidad debido a la escasez de tierra para cultivar. 


\section{BIBLIOGRAFÍA}

BARBEITO, A y LO VUOLO, R (1995): La Modernización Excluyente. Transformación Económica y Estado de Bienestar en Argentina. Buenos Aires, Losada S.A.

BESIL, Antonio C. (1969): Análisis de las causas del actual cambio en la estructura del sector agrícola de la provincia del Chaco. Tesis Doctoral. Resistencia, Chaco Facultad de Ciencias Económicas, UNNE.

BROWN, Harres B. y WARE, Jacob O(1961): Algodón. México, D.F. UTHEA,

BRUNIARD Enrique D. (1992): "El ámbito subtropical en la República Argentina" (Climatología dinámica y límites climáticos.). En: Estudios Geográficos $N^{\circ} 208$, Revista publicada por el Instituto de Economía y Geografía Aplicadas, Madrid, España.

BRUNIARD, Enrique D.(1978): El Gran Chaco Argentino. Ensayos de interpretación geográfica, GEOGRÁFICA 4, Resistencia, Chaco.

BRUNIARD, Enrique D.(1981): El Clima de las planicies del norte argentino (Tesis Doctoral). Resistencia, Chaco. Facultad de Humanidades UNNE.

BURGOS Juan J. (1970): "El clima de la Región Nordeste de la República Argentina en relación con la vegetación natural y el suelo". En: Boletín de la Sociedad Argentina de Botánica, Volumen $\mathrm{XI}$, Buenos Aires.

CARDOZIER, U. R (1962): Cultivo y producción del Algodón. México, D.F. Herrero.

CARLEVARI, Isidro J. F(1996): La Argentina: Estructura Humana y Económica. $2^{\circ}$ Edición, Buenos Aires, Macchi

CEIL, Adela (1991): De la agricultura tradicional a la tecnológica. Madrid. Cincel.

CIMMYT, (1993): Programa de economía. La adopción de tecnologías agrícolas: guía para el diseño de encuestas. México . D.F.

DAVID, Jean (1971): Le Coton et L'Industrie Conniere. Paris. Presses Universiteries de France.

DIARIO NORTE (1998): Sección Rural, junio 1998. Resistencia, Chaco.

FUNDACION PARA LA EDUCACION, LA CIENCIA Y LA CULTURA, (1988): El Deterioro del Ambiente en la Argentina (Suelo-agua-Vegetación-Fauna).Buenos Aires.

GELMAN, Susana (1995): "Análisis del Producto Bruto Geográfico de la Provincia del Chaco. En: Indicadores Económicos. Revista del Departamento de Finanzas de la Facultad de Ciencias Económicas de la UNNE, Año 4, № 22, Resistencia, Chaco.

ICAC RECORDER (1997). Información Técnica y Estadística del cultivo del Algodón. En formato Digital (CD-Room).

INSTITUTO DE GEOGRAFÍA de la Facultad de Humanidades de la UNNE (1989). Geográfica 6: Atlas Geográfico de la Provincia Chaco, Tomo I: La tierra y la Agricultura. Resistencia, Chaco.

INSTITUTO DE GEOGRAFÍA de la Facultad de Humanidades de la UNNE (1990). Geográfica 7: Atlas Geográfico de la Provincia Chaco, Tomo III: La Población y el Hábitat. Resistencia, Chaco.

INSTITUTO NACIONAL DE TECNOLOGÍA AGROPECUARIA (INTA) EERA de Presidencia Roque Sáenz Peña. Mapa de Capacidad de Uso del Suelo en el Chaco. Escala 1:500.000.

INTA (Instituto Nacional De Tecnología Agropecuaria) (1965): Guías prácticas agrometeorológicas. Publicación micelánea 34, Buenos Aires.

INTA: Noticias Página Web, Junio 1999.

KUGLER, Nicolás Diego (1994): Razones para migrar. Una estudio de población del Chaco Algodonero. Tesis de Licenciatura (Inédito). UBA. Buenos Aires.

LARGIÉRE, Robert (1968): El algodón. Técnicas agrícolas y producciones tropicales. Barcelona, Blume.

MANOILOFF, Raúl O.: El cultivo del algodón desde 1950. A al actualidad (la etapa de crisis) Inédito. Facultad de Humanidades. UNNE. Resistencia, Chaco.

MILLER Austin A. (1975): Climatología. Barcelona, Omega.

MIRANDA, Guido A (1955): Tres ciclos chaqueños. Crónica histórica regional. Resistencia, Chaco, Norte Argentino.

PEPE, Norberto C. y DEREWICKI, José V.(1997): Su majestad el Algodón. Resistencia, Chaco, Meana y Meana. 
POLAN, Lacki (1995): Buscando soluciones para la crisis del agro, ¿en la ventanilla del banco, o en el pupitre de la escuela?. Santiago de Chile.

RAMIREZ, Mirta L. (1996): Análisis y perspectivas de la situación agroproctiva y social de las provincias del Nordeste Argentino, Paraguay y Estados del Sur de Brasil. Tesis de Licenciatura (Inédito). Facultad de Humanidades.UNNE. Resistencia, Chaco.

ROCCATAGLIATA, J. A. (1988): La Argentina: geografía general y los marcos regionales. Buenos Aires, Argentina, Grupo Editorial Planeta Argentina.

ROFMAN. Alejandro B. (2000): Desarrollo Regional y Exclusión Social. Transformaciones y crisis en la Argentina contemporánea. Buenos Aires, Amorrortu editores.

SOCIEDAD ARGENTINA DE BOTÁNICA IX (1970): Jornadas Argentinas de Botánica. Volumen XI, Buenos Aires.

STRALHER, Arthur N.(1982): Geografía física. Barcelona, Omega.

VALEIRO, Alejandro (1998): El Nuevo Algodón ¿Son sustentables los cambios recientes del sector algodonero Argentino? Publicación del INTA, Santiago del Estero. 


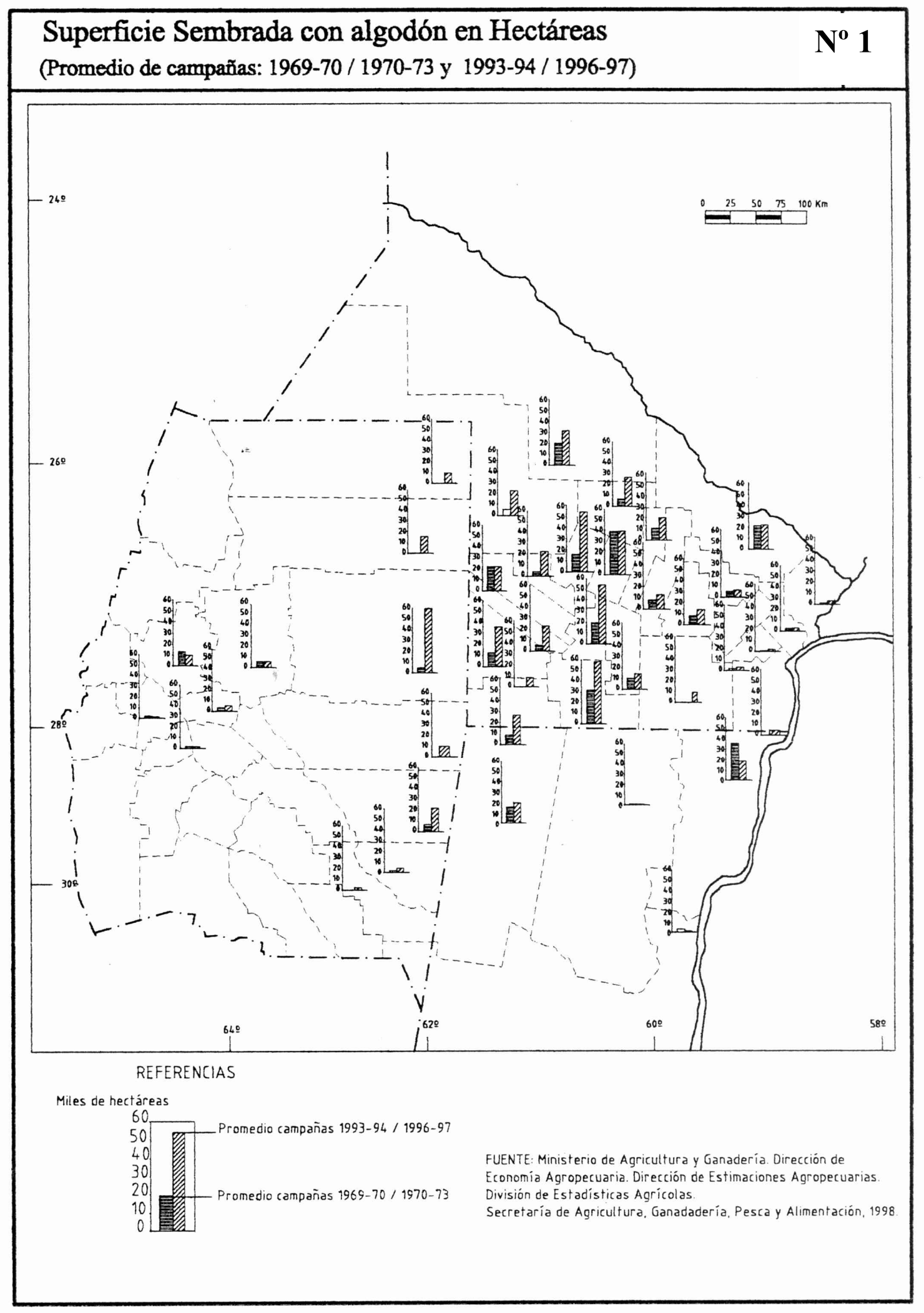




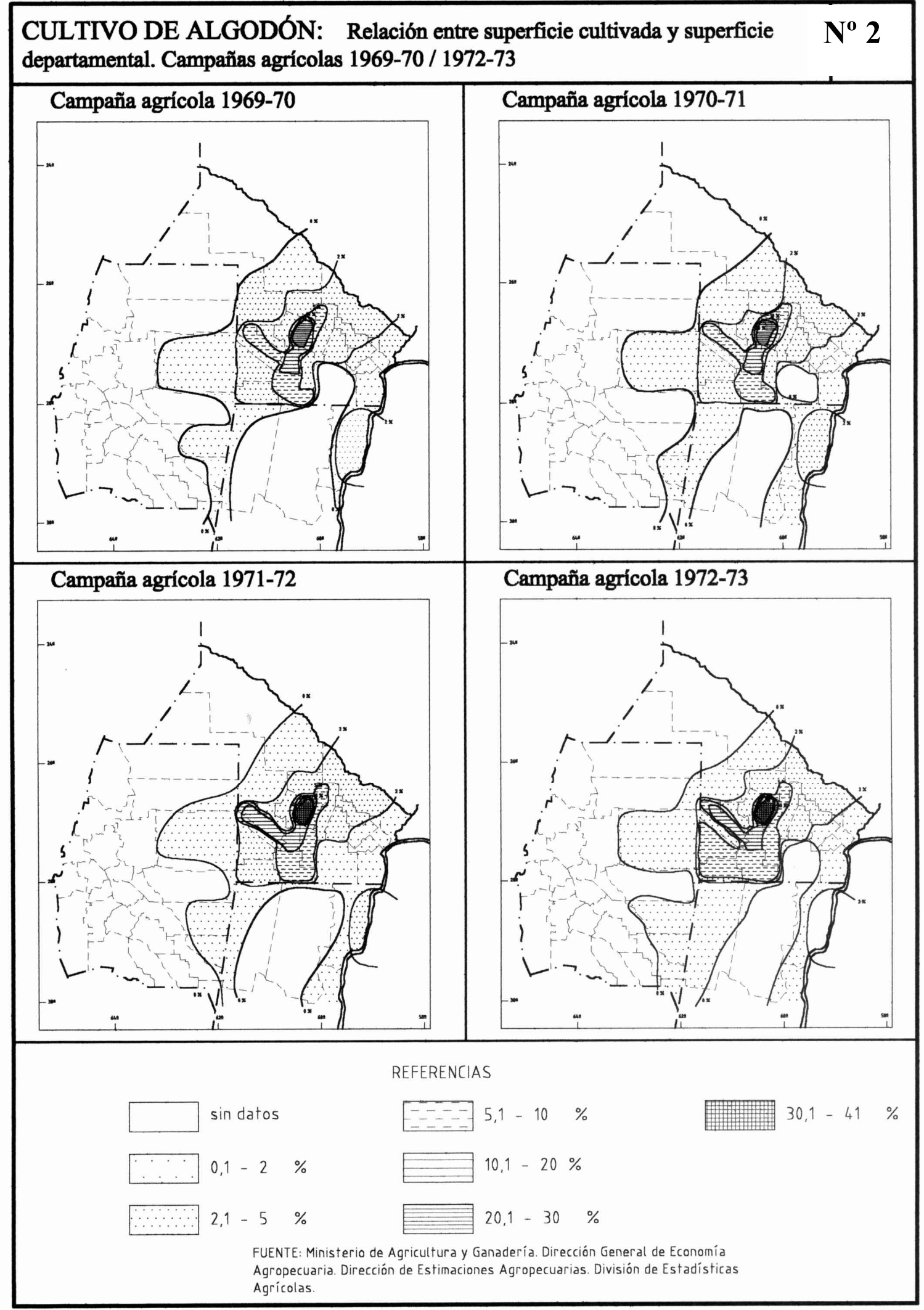




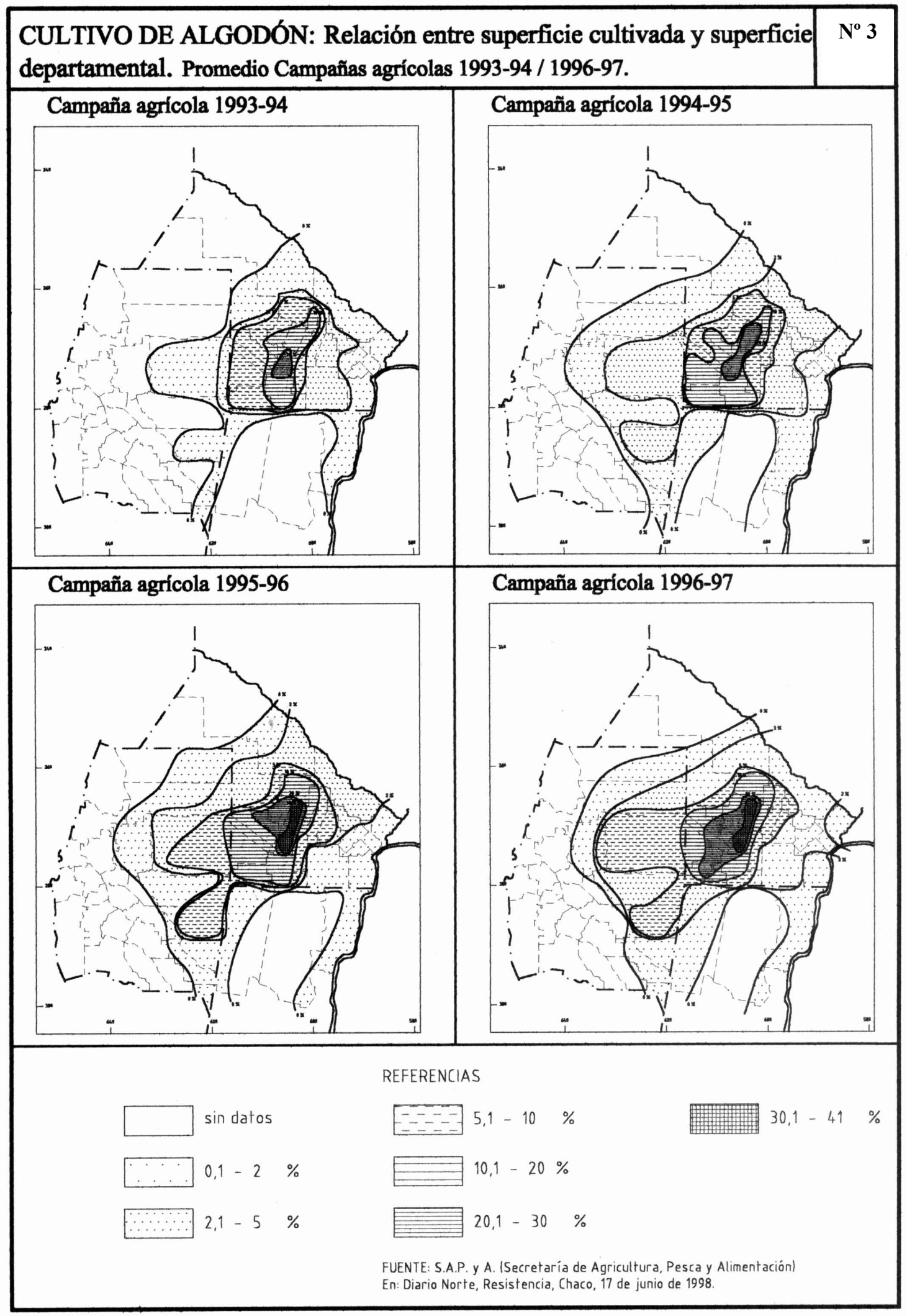

